\title{
Case Report 63
}

Ramiro Hernandez, M.D., Kathleen P. Heidelberger, M.D. and Andrew K. Poznanski, M.D.*

Division of Pediatric Radiology and Department of Pathology, The University of Michigan Medical Center, Ann Arbor, Michigan, USA
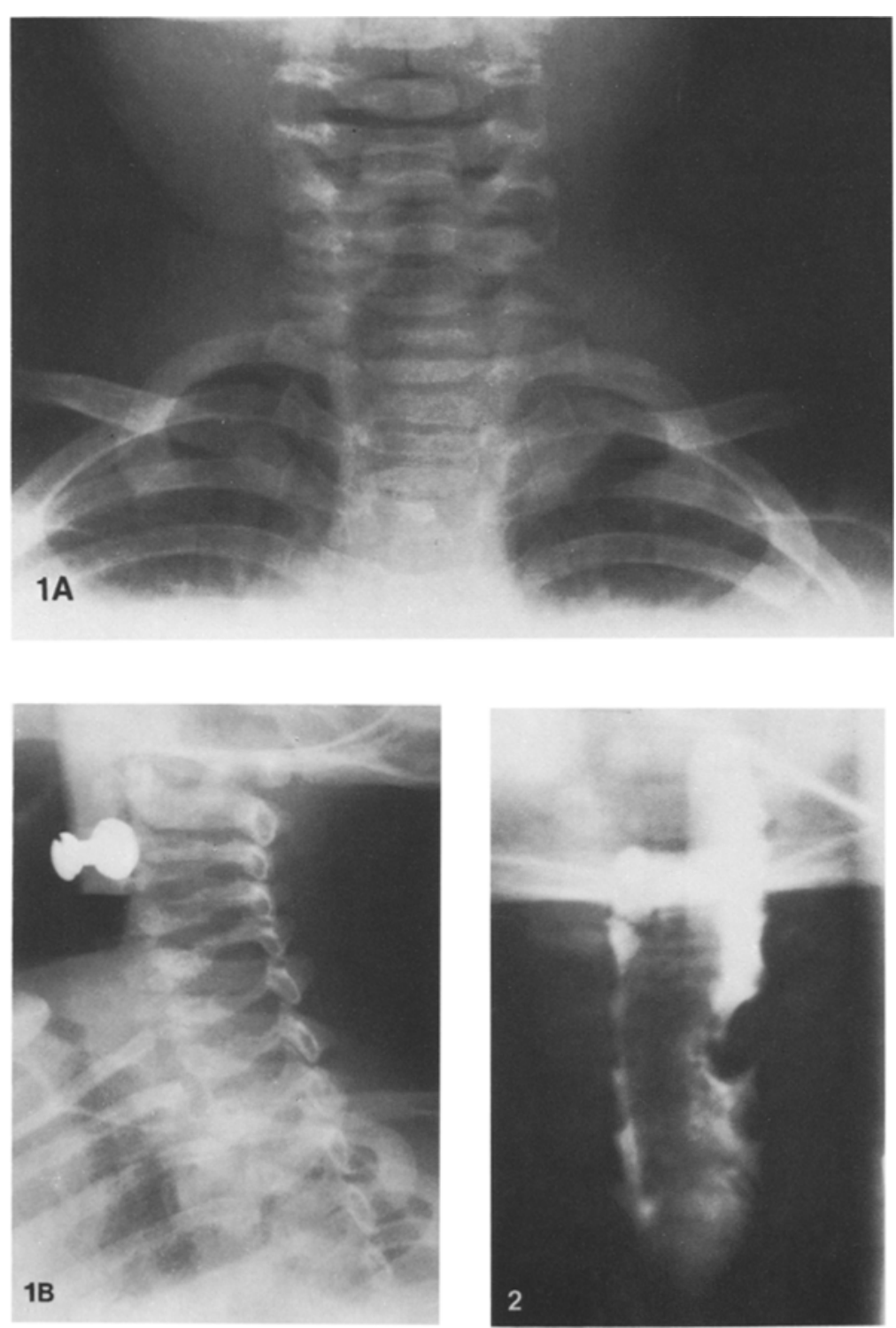

* Submitted for publication in October 1977

Address reprint requests to: R. Hernandez, M.D., Division of Pediatric Radiology, The University of Michigan Medical Center, Ann Arbor, MI 48104, USA
Fig. 1. A An anteroposterior view of the lower two-thirds of the cervical spine shows a soft tissue mass on the left side in the base of the neck and in the upper portion of the superior mediastinum of the thorax. Enlargement of the neural foramina of C- $-5-C-6$ and $C-6-C-7$ is present. The mass is not radiologically mineralized (an opaque density in the area of the mass is an artefact). B A left oblique view of the cervical spine shows more clearly the enlargement of the C-5-C-6 and C-6-C-7 intervertebral foramina

Fig. 2. An anteroposterior film obtained during a cervical myelogram shows a $12 \times 7 \mathrm{~mm}$ defect centered at the C-5-C-6 intervertebral foramen on the left. The mass has the appearance of an extradural lesion. A similar but smaller defect is noted at the C-6-C-7 level

\section{History}

An eight-month-old white male infant with an uneventful past history presented with weakness of the left upper extremity. The remainder of the physical examination was normal initially, but subsequent examination disclosed a firm, irregular nodular mass in the left side of the neck, attached to the deep structures. Progression of the weakness of the left arm, particularly distally, was observed in the following two months, with the development of a left Horner syndrome and an absent left biceps reflex. The electromyogram was compatible with a diffuse brachial plexus abnormality. Roentgenograms of the cervical spine were obtained (Figs. $1 \mathrm{~A}$ and $\mathrm{B}$ ) and a myelogram was performed (Fig. 2).

On surgical exploration a tumor was noted to involve the brachial plexus and subclavian artery. A biopsy was done.

The infant expired one month after the surgical exploration. An autopsy was not performed. 


\section{Histological Sections}
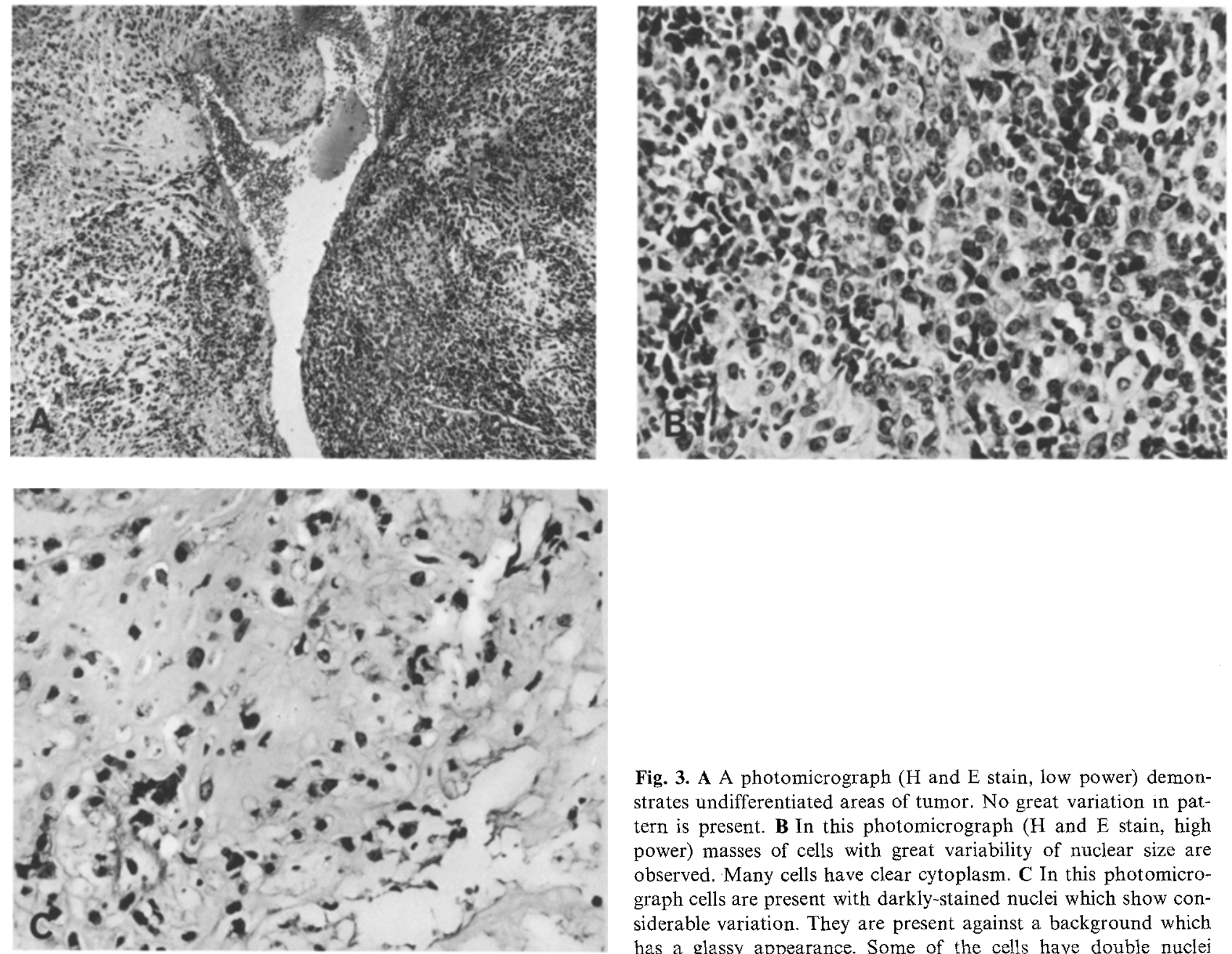

Fig. 3. A A photomicrograph ( $\mathrm{H}$ and $\mathrm{E}$ stain, low power) demonstrates undifferentiated areas of tumor. No great variation in pattern is present. B In this photomicrograph ( $H$ and $E$ stain, high power) masses of cells with great variability of nuclear size are observed. Many cells have clear cytoplasm. C In this photomicrograph cells are present with darkly-stained nuclei which show considerable variation. They are present against a background which has a glassy appearance. Some of the cells have double nuclei

\section{Diagnosis: Extraskeletal (Soft Tissue) Mesenchymal Chondrosarcoma of the Neck}

The differential diagnosis must include a large neurogenic tumor e.g. ganglioneuroma, neurofibroma, sarcomatous transformation of a nerve tumor, neuroblastoma, teratoma, rhabdomyosarcoma and other rare mesenchymal neoplasms.

\section{Discussion}

Mesenchymal chondrosarcoma, a rare malignant neoplasm which can be either skeletal or extraskeletal in origin, was originally described by Lichtenstein and Bernstein. In 1973, Guccion et al. collected 65 cases from the literature, of which 44 occurred in bone and 21 in extraskeletal tissues. The authors added 10 more cases, all of extraskeletal origin.

Clinically, extraskeletal mesenchymal chondrosarcoma occurs slightly more often in females than in males. More than half the patients are in the second and third decades of life. The soft tissue mesenchymal chondrosarcoma shows a predilection for the head and neck, although the tumor has been reported in the thigh, leg and thumb. These soft tissue neoplasms are infrequent in the trunk, but have been observed occasionally in the paraspinal muscles and in the chest wall.

The skeletal lesions are most commonly observed in the ribs, facial bones, calvaria and spine. They are relatively uncommon in the appendicular skeleton.

A painful soft tissue mass is often the presenting 
feature, both of the extraskeletal and skeletal types.

The pathological manifestations of extraskeletal mesenchymal chondrosarcoma are interesting. Typically, the lesion is a large, multilobulated, well-circumscribed tumor, with an average diameter of about $13 \mathrm{cms}$. The periphery of the mass tends to be soft and fleshy and the central portions firm, containing bluish-green nodules of cartilage and, occasionally, osteoid and bone. Necrosis and hemorrhage may be present.

Microscopically, a unique and distinctive histological pattern generally is noted, characterized by the presence of solid sheets of highly cellular, undifferentiated mesenchymal cells interspersed with islands of relatively well-differentiated cartilage. The mesenchymal cells contain large vesicular nuclei with prominent nucleoli, some of which are multiple. The presence of mitotic figures is variable. The mesenchymal cells are often observed to aggregate around vascular spaces, reminiscent of hemangiopericytoma.

Radiologically, in the extraskeletal variant, a poorly-defined, generally large, soft tissue mass is identified, which may contain irregular stippled or amorphous calcification, whose presence should suggest a lesion of chondroid origin. In the skeletal form the tumor is primarily lytic in appearance with varying degrees of aggressive behavior in the reported cases. In some instances the zone of transition around the lytic lesion in bone is narrow, even suggesting a benign neoplasm, while in others it is characteristically wide, indicating its aggressive nature.

The biological behavior of this neoplasm is somewhat difficult to assess, because adequate follow-up of reported cases is frequently lacking. Several of the cases reported proved to be highly malignant, with death occurring anywhere from eight months to five years after the diagnosis had been established. In other instances, a more protracted course was followed, with death coming on as late as eleven years after the diagnosis was made.

\section{References}

1. Guccion, J.G., Font, R.L., Enzinger, F.M., Zimmerman, L.E.: Extraskeletal mesenchymal chondrosarcoma. Arch. Pathol. 95, 336 (1973)

2. Lichtenstein, L., Bernstein, D.: Unusual benign and malignant chondroid tumors of bone. A survey of some mesenchymal cartilage tumors and malignant chondroblastic tumors, including a few multicentric ones, as well as many atypical benign chondroblastomas and chondromyxoid fibromas. Cancer 12, 1142 (1959)

3. Salvador, A.H., Beabout, J.W., Dahlin, D.C.: Mesenchymal chondrosarcoma-observations on 30 new cases. Cancer 28, 605 (1971) 